\title{
The ASTRI Dual-Mirror Small-Size Cherenkov Telescope
}

\section{Cornelia Arcaro*}

Centre for Space Research, North-West University, Potchefstroom 2520, South Africa and INAF

Osservatorio Astronomico di Padova, vicolo dell'Osservatorio 5, 35122, Padova, Italy

E-mail: cornelia.arcaro@nwu.ac.za

for the CTA ASTRI project

The ASTRI dual-mirror small-size telescope (ASTRI-Horn) is an imaging atmospheric Cherenkov telescope that was developed by the Italian National Institute of Astrophysics (INAF) within the framework of the Cherenkov Telescope Array (CTA) project, the next-generation ground-based very-high-energy gamma-ray observatory. The CTA observatory will consist of two sites configured in two arrays of telescopes of different sizes. They will be located in the Northern and Southern Hemispheres to cover the full sky. ASTRI (Astrofisica con Specchi a Tecnologia replicante Italiana) is the Italian proposal for the small-size telescopes and its design is based on a Schwarzschild-Couder configuration. To fulfill the required plate scale, the camera is based on a new technology involving the use of Silicon-photomultipliers. With its wide field of view of 10 degrees, it is optimized for gamma-ray observations in the range from few $\mathrm{TeV}$ up to several tens of TeV. The ASTRI prototype is installed at Serra La Nave at $1735 \mathrm{~m}$ a.s.l. in Sicily, Italy, and is currently going through the science validation phase. A further development of the program foresees the construction of a set of nine ASTRI telescopes proposed as pathfinder for the CTA array. In this contribution, the main features of the ASTRI telescope will be described and an update on the latest news on the prototype activities and the mini-array will be presented.

High Energy Astrophysics in Southern Africa - HEASA2018

1-3 August, 2018

Parys, Free State, South Africa

\footnotetext{
* Speaker.
} 


\section{Introduction}

Being the most sensitive instruments to very-high-energy (VHE, $E>50 \mathrm{GeV}$ ) gamma rays, ground-based imaging atmospheric Cherenkov telescopes (IACTs; e.g., see [1] for a review) currently investigate this energetic radiation. They exploit the Earth's atmosphere as absorbing medium of a calorimeter and detect the cascade of secondary particles. Such particles are produced during the absorption of incoming VHE gamma rays. IACTs are most effectively operated when several of these are set apart by $\sim 100 \mathrm{~m}$ and pointed to the same sky position. In this way, they observe the same shower with different orientation, which allows its stereoscopic imaging and reconstruction. The Cherenkov Telescope Array [2] represents a next generation of IACTs with unprecedented sensitivity, spectral coverage, angular and energy resolution and survey capabilities in order to significantly expand the science possibilities in the VHE range, in particular at the lowest and highest energies detectable. CTA is an observatory that will consist of two arrays with several tens of IACTs of different sizes located at two sites, one in the northern and another in the Southern Hemisphere, to provide full sky coverage. While the northern site will be located on La Palma at the Canary Islands, the southern site is proposed to be located in the Atacama Desert in Chile.

Aiming at an energy coverage spanning from $20 \mathrm{GeV}$ to at least $300 \mathrm{TeV}$, the southern CTA array will consist of three different telescope types, which are referred to as large-, medium- and smallsized telescopes respectively (LSTs, MSTs and SSTs). While LSTs provide sensitivity at the lowest energies $(20<E<150 \mathrm{GeV})$, SSTs are optimised to the highest VHE energies $(5<E<300 \mathrm{GeV}$; [3]). There are currently three different SST prototypes under development within the CTA Collaboration, i.e., the single-mirror SST [4] and the dual-mirror (2M) telescopes GCT [5] and ASTRI [6].

The latter is conducted by the Italian National Institute for Astrophysics (INAF) since 2011 with the aim to design and develop within the CTA framework an end-to-end prototype of the SST in $2 \mathrm{M}$ configuration. While the activities regarding the science validation of the prototype are under completion, INAF approved the production of a set of nine ASTRI-like telescopes and proposed them as pathfinder for the CTA array in the south site. The pathfinder will help in the technical implementation of the CTA array but also produce science data [7].

\section{The ASTRI-Horn Telescope}

After its installation at the INAF "M.C. Fracastoro" observing station at Serra La Nave (Mount Etna, Sicily), the ASTRI prototype telescope (see figure 1) was inaugurated in September 2014. On 2018, November 10th, the ASTRI prototype was dedicated to Guido Horn d'Arturo, the Italian astronomer who first conceived and realized a telescope with a large mirror made of segments. Now the ASTRI dual-mirror telescope prototype is simply named ASTRI-Horn. It is currently tested and commissioned under field conditions and entered recently the scientific verification phase.

The ASTRI-Horn telescope is based on the so-called Schwarzschild-Couder (SC) design proposed by Vassiliev et al. [8] for the application in Cherenkov astronomy, allowing to correct contemporaneously spherical, coma and astigmatism aberrations on a large field of view. It is a compact system, with a $4 \mathrm{~m}$-diameter primary and a $1.8 \mathrm{~m}$-diameter secondary mirror, with a primary-to-secondary distance of $3 \mathrm{~m}$, and a secondary-to-camera distance of $0.52 \mathrm{~m}$ [9]. It adopts 


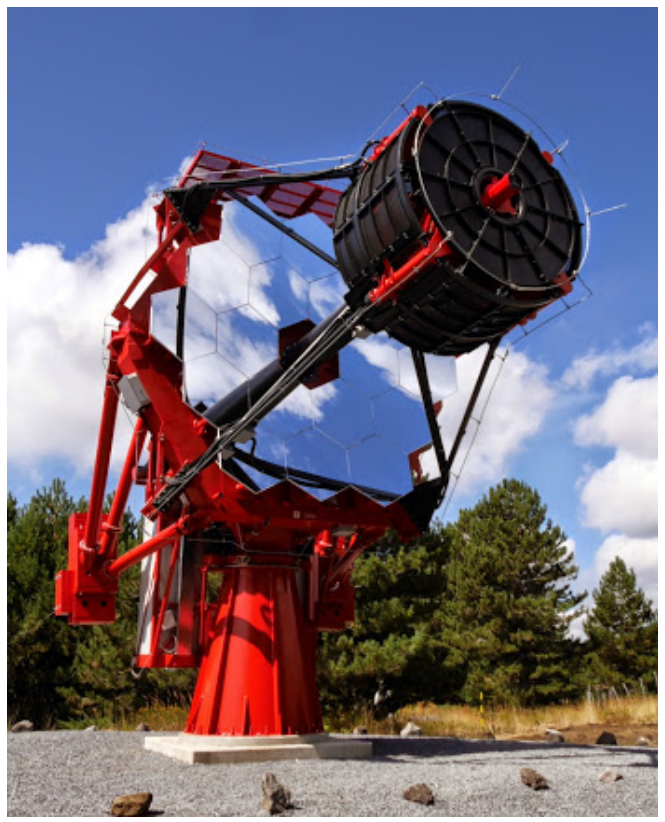

Figure 1: The ASTRI-Horn dual-mirror telescope located at the INAF "M.C. Fracastoro" observing station at Serra La Nave.

an altitude-azimuthal design, with the mirror dish being mounted on the azimuth fork.

The aspherical optical profile of the primary mirror is approximated by 18 hexagonal spherical glass segments, which are divided into three groups with different nominal radius of curvature, while M2 is a monolithic glass substrate of $19 \mathrm{~mm}$ thickness. While the segments of the primary-mirror are obtained via cold slumping [10], the secondary mirror is produced by means of hot slumping [11]. Moreover, the primary-mirror segments have a sandwich-like structure, with an area density of about $12 \mathrm{~kg} / \mathrm{m}^{2}$, making them light weight such that handling and mounting operations are easy.

The ASTRI-Horn telescope design includes a novel camera design that exploits the characteristics of the telescope's SC design, leading to a very compact camera $(0.52 \times 0.66 \times 0.56 \mathrm{~mm}$; [12]). The fundamental elements of this novel design are the choice of photosensor, i.e., Silicon photomultipliers (SiPMs), and the read-out electronics.

With linear dimensions of few millimeters, SiPMs are suited to match to the point-spread function (PSF) of ASTRI. In addition, they exhibit high quantum efficiency in the near ultraviolet wavelength range where the Cherenkov light is maximum ( $>35 \%$ at $400 \mathrm{~nm}$ ), very fast response, and excellent single photoelectron (phe) resolution. The camera pixel have a size of $7 \times 7 \mathrm{~mm}$ corresponding to $0.19^{\circ}$ on the sky.

Being based on a custom peak-detector operation mode for the acquisition of the SiPM pulses, the read-out electronics represent an innovative solution that is based on a CITIROC ASIC (application specific integrated circuit; [13]). The signal shaper and the peak detector, which are customized for ASTRI, provide high efficiency, auto-trigger capability and very fast pixel read out. The camera trigger is topological, meaning it is activated when a given number of contiguous pixels shows a signal above a certain threshold equivalent to a given number of photoelectrons, with both the 
number of contiguous pixels and the number of photoelectrons being adjustable. A particular operating mode of the read-out electronics is the so-called variance technique [12,13], for which the electric signal generated by each pixel that is not triggered by the first level trigger is continuously sampled. While the sequence of ADC (Analog to Digital Converter) values obtained is constant with time, its variance is proportional to the photon flux impinging on the pixel. The acquisition of the variance data is performed in parallel to the normal data acquisition. The method has several applications ranging from validating the pointing accuracy of the telescope and determining the Night Sky Background (NSB) to monitoring the mirror alignment.

\section{The commissioning of the ASTRI-Horn telescope}

Since its inauguration in 2014, an extensive set of tests was performed, to characterize and subsequently monitor the performance of the ASTRI-Horn telescope. While the validation phase for the mechanical structure and for the optics has been concluded, the camera characterization is still ongoing.

The validation of the optical design has been conducted during a dedicated campaign in Autumn 2016. The optics' PSF has been determined as a function of the position along the telescope's field of view [14]. In addition, the temporal stability of the PSF was determined in function of the telescope elevation [9]. The measurements were carried out with a CCD camera placed at different positions in the telescope's focal plane imaging stars at different sky positions showing that the required specification of a flat PSF of $\sim 10$ arcminutes along a large field of view $\left(\sim 10^{\circ}\right)$ is fulfilled. The PSF stability with elevation and with time [9] has a huge impact on the design of the active mirror control, allowing to use this control as an AIV (Assembly-Integration-Verification) tool for the alignment of the primary mirror's segments at the time of installation and whenever required (typically once per year).

The monitoring of the optic's reflectivity is crucial for the degradation evaluation of the reflective mirror coating due to the environmental impact by atmosphere, dust, sand, UV light, etc. The degradation rate is an important parameter with an impact on maintenance operations of the mirrors, implying for example the necessity of an on-site recoating facility. For this reason, the reflectivity of the primary and secondary mirrors has been measured at different time scales. The measurements showed that the ageing of the coating is faster than expected, especially for the secondary mirror, which is most likely due to the aggressive volcanic atmosphere with frequent emission of sulphur compounds [15]. An identical coating was used for the glass mirrors of the MAGIC telescopes that are located on Roque the los Muchachos on La Palma [16]. In more than 8 years, the degradation of the reflectivity has been a few percent [17]. As the southern site chosen for CTA has no volcanos in the immediate environment and no aggressive atmosphere, the rate of degradation should be in line with CTA requirements.

The electromechanical structure has been intensively studied and its parameters have been optimized to achieve the maximum performance as thoroughly described in [6]. As part of regularly scheduled activity, an observational campaign to monitor the tracking and pointing performance as well as the astrometric precision of the telescope between November 2017 and January 2018 has been carried out. The measurements were obtained using the pointing monitoring camera (PMC), an auxiliary CCD camera placed at the back of the secondary mirror-supporting structure whose 
main purpose is to calibrate the pointing position of the telescope in the sky. During the test campaign, the telescope was either pointing to a fixed position or was tracking astronomical objects such as stars. The images were acquired at a frequency of one every 10 seconds, with exposure times typically of the order of few seconds and durations of an observational run between 10 and 20 minutes.

First, the astrometry [18] was computed for images taken during various pointings, revealing ASTRI's astrometric precision of 5 arcseconds RMS to meet the CTA requirements. Similarly, the tracking precision was determined from the PMC images acquired during several trackings, indicating a typical precision $\sim 6$ arcseconds RMS, thus meeting the CTA specifications on tracking precision. The pointing precision was evaluated using an in-house developed IDL-based code according to [19], being of the order of $\sim 20$ arcseconds.

The integration of the ASTRI camera at the telescope was carried out in May 2017, and despite the fact that the camera performance was far from optimum the telescope detected first Cherenkov light [20]. Since then, the camera underwent engineering tests, and some scientific runs were performed. First trigger rates have been recorded as function of the pixel trigger thresholds during moonless nights while pointing the telescope at different sky positions, with a topological trigger of at least five contiguous pixels $[12,21]$. A preliminary analysis of the curves indicated the camera's working point to be at $\sim 11$ phe, meaning that the trigger rate in the region dominated by the air shower events is lower than expected from measurements previously obtained in lab. This behavior indicates a reduced telescope efficiency, that is most likely associated with mirror degradation and misalignment, which were further investigated. On the other hand, the trigger threshold was found to be higher [12] due to a local NSB higher than expected.

In December 2017, 1.7 hours of gamma-ray data on the Crab Nebula and 1.4 hours of dedicated off target data were collected, respectively, resulting in a non-detection (see [21] for details) of this well-known VHE emitter. However, given the limited exposure time, the reduced efficiency of the telescope, the non-optimal tuning of the camera, and the rough calibrations used for the data reduction, this was not unexpected. Nevertheless, these observations allowed to verify the end-toend concept of the prototype, from the photon detection to the data analysis, with a complete data analysis report being given in [21].

The variance mode has been evaluated in January and March 2018 and showed its potential in assessing the telescope's pointing behavior [10] and checking the optical alignment of the M1 segments [12]. Indeed, the variance data indicated some misaligned M1 mirrors. These findings were confirmed in May when six mirrors were resulted to be misaligned and thus needed to be realigned subsequently. For two mirrors the misalignment was not revealed by the variance data, since they were yet aligned very close to their nominal position and thus not visible in the variance data. Two considerations can be deduced: first, the optical alignment is quite stable in time as this is the first time in almost two years that an adjustment of the M1 mirrors was required, and second the variance technique is an efficient method to monitor the optical alignment of the M1 mirrors. The analysis of the variance data regarding their potential to assess the pointing behavior of the telescope is still ongoing. 


\section{The ASTRI mini-array}

\subsection{Technological and operational development}

The next step of the program is the production of a set of nine end-to-end ASTRI telescopes named ASTRI mini-array based on the ASTRI-Horn telescope design and proposed to be installed as pathfinder at the CTA observatory (CTAO) southern site. The development of the ASTRI miniarray has been approved by INAF in synergy with the Universidade de Sao Paulo (Brazil) and the North-West University (South Africa).

The pathfinder is proposed as a precursor of CTA to prove the technology and to test operational and maintenance procedures. In particular, the activities in which the pathfinder can give a fundamental contribution to the development of the CTAO southern site are: the assessment of the array trigger; the test of the array control software and general operations; the test of the assembly, integration and verification procedures on site (time, personnel, infrastructure, and tools); the test of the array performance against Monte Carlo simulations and of the implementation of the intensity interferometry capability.

The implementation of the pathfinder will happen within the framework of CTAO development. In particular, it will have to pass before deployment all CTAO technical reviews. For those ASTRI subsystems for which the design is already well consolidated, i.e., the mirror elements and the camera read-out electronics, the production has already started, with a minimal modification of the mirror coating in order to enhance the reflectivity in the wavelength range of interest specified by CTA requirements [15] and to lower the contribution of the NSB in the infrared, while keeping the same performance in terms of resistance and durability.

\subsection{Science with the ASTRI mini-array}

Besides the technological and operational aspects, the ASTRI mini-array is designed to perform astrophysical observations. Although the sensitivity is worse than that of the full CTA southern-site SST array [2], the energy and angular resolutions are in line with those of the 70 SST telescope array since only a few $\mathrm{TeV}$ shower events are expected to trigger more than nine units, resulting an energy threshold of 10-15\%. Based on preliminary Monte Carlo studies performed with the A-SciSoft package [22], the ASTRI mini-array could push the sensitivity of the current generation of IACTs further above $10 \mathrm{TeV}$, extending up to about $100 \mathrm{TeV}$. Several studies have been carried out to simulate the $\mathrm{TeV}$ gamma-ray emission of promising sources accessible to the ASTRI mini-array to evaluate its potential science cases.

The ASTRI mini-array will exploit its sensitivity and extended spectral range to investigate the emission of prominent sources with hard spectra such as galactic sources like young ( $\sim 2000$ years) pulsar wind nebulae (e.g., Crab Nebula and Vela-X) and supernovae remnants (SNRs; e.g., Velajunior and RX J1713.7-3946). Being optimised for the highest energies and featuring a large field of view in combination with a good angular resolution, the ASTRI mini-array could contribute to the understanding of the origin of cosmic rays (CR) by distinguishing between hadronic and leptonic emission scenarios for this kind of sources. More important, by observations of the highenergy tail of the spectra of VHE emitting sources located at the Galactic Centre, the mini-array can investigate the existence of cosmic rays with energies of the order of $\sim 10^{15} \mathrm{eV}$, so-called PeVatrons, as they seem to have been observed in Sagittarius A* by H.E.S.S. [24]. 
Another promising target for the ASTRI mini-array are extragalactic sources with hard spectra, as for example extreme blazars like 1ES 229+200 in order to shed light on the mechanism of gammaray emission that may eventually involve ultra-high-energy cosmic rays (UHECRs). In the case of 1ES 229+200, a gamma-ray emission above $25 \mathrm{TeV}$ would imply hadronic emission mechanisms involving the acceleration of UHECRs.

A cosmological study closely related to this type of sources of potential UHCRS emission and to their hard emission spectra involves anomalies in the opacity of the Universe to VHE gamma rays due to the extragalactic background light (EBL) by the existence of axion-like particles (ALPs) and aims at constraining the intergalactic magnetic field (IGMF). The science case is based on an oscillation of a gamma ray to an ALP in the IGMF, thus travelling to the Earth without being absorbed by the EBL. In the Earth's magnetic field, the ALPs transform back to gamma rays that can be observed by the ASTRI mini-array. In addition to this, EBL studies at wavelengths $>10 \mu \mathrm{m}$ can be carried out by observing near-by so-called high-frequency-peaked BL Lac objects (e.g., Mrk 421 and Mrk 501) and radio galaxies (e.g., M 87), where the EBL absorption only kicks in at $\sim 30 \mathrm{TeV}$.

\section{Conclusion}

CTA represents the next generation of ground-based observatories for gamma-ray astronomy at very-high energies, becoming the world's largest and most sensitive gamma-ray observatory. The southern-site CTA array will consist of three different telescope types, that is the Large-, Medium-, and Small-Sized Telescopes (LSTs, MSTs and SSTs). Since its installation on the Etna at Sicily, the ASTRI dual-mirror telescope, a prototype of the SST class for CTA, has been extensively studied, and it has been demonstrated to fulfill expectations and requirements. The camera commissionning and the science verification phase is still ongoing, while the next phase towards the implementation of a mini-array of nine ASTRI telescopes has already been entered. The ASTRI mini-array is proposed as one of the first building blocks of the CTAO southern site and will allow early science operations extending the observation of gamma rays in an energy range previously unexplored by current IACT facilities.

\section{Acknowledgement}

This work is supported by the Italian Ministry of Education, University, and Research (MIUR) with funds specifically assigned to the Italian National Institute of Astrophysics (INAF) for the Cherenkov Telescope Array (CTA), and by the Italian Ministry of Economic Development (MISE) within the "Astronomia Industriale" program. We acknowledge support from the Brazilian Funding Agency FAPESP (Grant 2013/10559-5) and from the South African Department of Science and Technology through Funding Agreement 0227/2014 for the South African Gamma-Ray Astronomy Programme. We gratefully acknowledge support from the agencies and organizations listed under Funding Agencies at this website: http://www.cta-observatory.org/.

\section{References}

[1] Rieger, F.M., de Ona-Wilhelmi, E., and Aharonian, F. A., TeV Astronomy, Frontiers of Physics, Volume 8 Issue 6 (2013), 714 [DOI:10.1007/s11467-013-0344-6] 
[2] Hofmann, W., The Cherenkov Telescope Array: Exploring the Very-high-energy Sky from ESO's Paranal Site, The Messenger, 168 (2017), 2

[3] The Cherenkov Telescope Array Consortium, Science with the Cherenkov Telescope Array, eprint [arXiv:1709.07997]

[4] Heller, M., et al., The Single Mirror Small Size Telescope of the Cherenkov Telescope Array, AIP Conference Proceedings, Volume 1792 Issue 1 (2017), ID 080003 [DOI:10.1063/1.4969024]

[5] Sol, H., Greenshaw, T., Le Blanc, O., and White, R., Observing the sky at extremely high energies with the Cherenkov Telescope Array: Status of the GCT project, in proceedings of the 35th International Cosmic Ray Conference (ICRC2017), [arxiv:1709.09134]

[6] Canestrari, R., et al., for the CTA ASTRI Project, ASTRI SST-2M prototype for the Cherenkov Telescope Array: status after the commissioning phase of the telescope, in proceedings of SPIE conferences, 10399 (2017), ID 1039904 [DOI:10.1117/12.2276767]

[7] Pareschi G., et al., for the ASTRI Collaboration and the CTA Consortium, The ASTRI SST-2M prototype and mini-array for the Cherenkov Telescope Array (CTA), in proceedings of SPIE conferences, 9906 (2016), ID 99065T [DOI:10.1117/12.2232275]

[8] Vassiliev, V., Fegan, S., and Brousseau, P., Wide field aplanatic two-mirror telescopes for ground-based g-ray astronomy, Astroparticle Physics, 28 (2007), 10 [DOI:10.1016/j.astropartphys.2007.04.002]

[9] Canestrari, R., et al., for the ASTRI Collaboration and the CTA Consortium, The ASTRI SST-2M prototype for the Cherenkov Telescope Array: opto-mechanical performance, in proceedings of SPIE conferences, 9906 (2017), ID 990619 [DOI:10.1117/12.2232270]

[10] Canestrari, R., et al. 2013, Cold-shaping of thin glass foils as novel method for mirrors processing. From the basic concepts to mass production of mirrors, Optical Engineering, 52 (2013), ID 051204 [DOI:10.1117/1.OE.52.5.051204]

[11] Ghigo, M., et al., Development of hot slumping technique and last optical performances obtained on a $500 \mathrm{~mm}$ diameter slumped segment prototype for adaptive optics, in proceedings of SPIE conferences, 7439 (2009), ID 74390 [DOIR:10.1117/12.826405]

[12] Catalano, O., et al., for the CTA ASTRI Project, The ASTRI camera for the Cherenkov Telescope Array, in proceedings of SPIE conferences, 10702 (2018), ID 1070237 [DOI:10.1117/12.2314984]

[13] Sottile, G., et al., for the ASTRI Collaboration and the CTA Consortium, ASTRI SST-2M Camera Electronics, in proceedings of SPIE conferences, 9906 (2016), ID 99063D [DOI:10.1117/12.2232464]

[14] Giro E., et al., First optical validation of a Schwarzschild Couder telescope: the ASTRI SST-2M Cherenkov telescope, Astronomy \& Astrophysics, 608 (2017), A86 [DOI:10.1051/0004-6361/201731602]

[15] Scuderi, S., et al., for the CTA ASTRI Project, From the Etna volcano to the Chilean Andes: ASTRI end-to-end telescopes for the Cherenkov Telescope Array, proceedings of SPIE conferences, 10700 (2018), ID 107005Z [DOI:10.1117/12.2312453]

[16] Pareschi, G., et al., Glass mirrors by cold slumping to cover $100 \mathrm{~m} 2$ of the MAGIC II Cherenkov telescope reflecting surface, in proceedings of SPIE conferences, 7018 (2008), ID 70180W [DOI:10.1117/12.790404]

[17] Razmik, M., et al., Absolute reflectance of a concave mirror used for astro-particle physics experiments, Astroparticle Physics, 105 (2018), 1 [DOI:10.1016/j.astropartphys.2018.09.001] 
[18] Lang et al., Astrometry.net: Blind astrometric calibration of arbitrary astronomical images, AJ, 139 (2010), 1782, [DOI:10.1088/0004-6256/139/5/1782]

[19] Wallace, P. T., TPOINT - Telescope Pointing Analysis System, Starlink User Note, 100 (1994)

[20] CTA Press Release, CTA Prototype Telescope, ASTRI, Achieves First Light, 14 June 2017

[21] Lombardi, S., et al., for the CTA ASTRI Project, ASTRI data reduction software in the framework of the Cherenkov Telescope Array, in proceedings of SPIE conferences, 10707 (2018), ID 107070R [DOI:10.1117/12.2311293]

[22] Pareschi G., Bonnoli, G., and Vercellone, S., for the ASTRI Collaboration and the CTA Consortium, The mini-array of ASTRI SST-2M telescopes, precursors for the Cherenkov Telescope Array, Journal of Physics: Conference Series, 718 (2016), ID 052028 [DOI:10.1088/1742-6596/718/5/052028]

[23] Burtovoi, A., et al., for the ASTRI Collaboration and the CTA Consortium, Prospects for PWNe and SNRs science with the ASTRI mini-array of pre-production small-sized telescopes of the Cherenkov telescope array, in AIP Conference Proceedings, 1792 (2017), ID 080007 [DOI:10.1063/1.4969028]

[24] Bonnoli, G., et al., for the ASTRI Collaboration and the CTA Consortium, Science with the ASTRI mini-array for the Cherenkov Telescope Array: blazars and fundamental physics, Journal of Physics: Conference Series, 718 (2916), [ID 052004]

[25] Abramowski et al., for the HESS Collaboration, Acceleration of petaelectronvolt protons in the Galactic Centre, Nature, 532 (2016), [DOI:10.1038/nature17147] 